\title{
IMPACT OF STATIC NODES AND PAUSE TIME ON THE STABILITY OF CONNECTED DOMINATING SETS IN A MOBILE AD HOC NETWORK
}

\author{
Natarajan Meghanathan \\ Jackson State University, 1400 Lynch St, Jackson, MS, USA
}

\begin{abstract}
The topology of mobile ad hoc networks (MANETs) change dynamically with time. Connected dominating sets (CDS) are considered to be an effective topology for network-wide broadcasts in MANETs as only the nodes that are part of the CDS need to broadcast the message and the rest of the nodes merely receive the message. However, with node mobility, a CDS does not exist for the entire duration of the network session and has to be regularly refreshed (CDS transition). In an earlier work, we had proposed a benchmarking algorithm to determine a sequence of CDSs (Maximum Stable CDS) such that the number of transitions is the global minimum. In this paper, we analyze the impact of pause time and static nodes on the performance (CDS Lifetime and CDS Node Size) of the Maximum Stable CDS and degree-based CDS. Pause time refers to the time a node stops at a location before moving again. When a node is declared to be a static node, it does not move at all during a simulation. We conduct the simulations by varying the maximum node velocity, the pause time of the mobile nodes and the percentage of static nodes. As we increase the maximum velocity with which any node could move, we observe to have greater chances of increasing the lifetime of the CDSs (especially the degree-based CDS) by letting all the nodes to move, but pause for an appreciable amount of time every now and then, rather than letting a certain a fraction of the nodes to remain static all the time, but have the rest of the nodes to move all the time.
\end{abstract}

\section{KEYWORDS}

Mobile Ad hoc Networks, Connected Dominating Set, Stability, Pause Time, Static Nodes, Node Degree, Simulations

\section{INTRODUCTION}

A mobile ad hoc network (MANET) is a dynamic distributed system of wireless nodes that move arbitrarily with time [1]. The wireless nodes operate in a limited transmission range and are battery-charged. Two nodes can communicate directly only if they are within the transmission range of each other. Hence, communication between any two nodes in a MANET is typically through one or more intermediate nodes (multi-hop paths). Several communication protocols have been proposed for unicast [2-3], multicast [4-5] and broadcast [6-7] communication in MANETs. In this paper, we focus on broadcast communication in MANETs. Specifically, our focus is on connected dominating sets (CDS), typically considered the graph-theoretic equivalent for a communication topology that can facilitate network-wide broadcasts. A CDS is a subset of the nodes in the network such that every node in the network is either in the CDS or is a neighbour (i.e., has a wireless link) of a node in the CDS. 
Broadcasts are considered to be resource-intensive operations in terms of both energy consumption at the nodes as well as the volume of traffic generated due to redundant retransmissions. If each node in the network broadcasts the message exactly once in its neighborhood, then every node receives a copy of the message from each of its neighbors (referred to as flooding). Though flooding guarantees that the broadcast message reaches every node in the network, each node loses energy to receive message broadcast by each of its neighbors (in addition to the energy lost at each node to transmit/ broadcast the message once in its neighborhood). It is not required for every node in the network to broadcast the message in its neighborhood. Instead, if we use a CDS like communication topology for broadcast, then it would be sufficient if only the constituent nodes of the CDS broadcast (retransmit) and the rest of the nodes only spend energy to merely receive the message. The lower the number of nodes constituting the CDS (referred to as CDS Node Size), the lower the number of retransmissions. Unfortunately, the problem of determining a minimum node size CDS is NP-hard [8]. Several heuristics have been proposed to determine CDSs with approximation ratios for the CDS Node Size as low as possible. A common thread among all of these heuristics is to give preference to include nodes that have a larger degree as part of the CDS so that the CDS Node Size is as low as possible. Apparently, nodes with a larger degree (larger number of neighbors) were the preferred candidates for inclusion to the CDS.

In [9], we observed that the degree-based heuristics are quite unstable in the presence of node mobility in MANETs. We had then proposed a benchmarking algorithm to determine a sequence of connected dominating sets over the duration of a network session such that the number CDS transitions is the global minimum. Referred to as the Maximum Stable CDS algorithm, the benchmarking algorithm assumes the entire sequence of future topology changes is known a priori and determines a sequence of long-living stable CDSs as follows: Whenever a CDS is required at time instant $t$, we determine a connected graph of the network (called a mobile graph) whose constituent edges exist for the longest possible time starting from time instant $t$; we then simply run a CDS algorithm/heuristic on the mobile graph and use the CDS for the duration of the mobile graph and repeat the above procedure for the entire network session. The algorithm can be used to arrive at a sequence of long-living stable CDSs such that the number of transitions (changes from one CDS to another) required during a network session is the global minimum and the average CDS lifetime for the network session is the global maximum (benchmarks).

As in many MANET simulation studies, the performance comparison of the Maximum Stable CDS proposed in [9] vis-a-vis the Degree-based CDS was only conducted when all the nodes in the network are mobile. In general, MANET simulation studies either consider the nodes to be mobile all the time or let the mobile nodes to pause for awhile every now and then [11-12]. To the best of our knowledge, no MANET simulation study has been conducted by considering a certain percentage of the nodes in the network to be permanently static and at the same time allowing the mobile nodes to pause for awhile every now and then. Hence, there is no idea regarding which of the two parameters (the \% of static nodes or pause time) would contribute towards a more stable network-wide communication topology like the CDSs. Also, it is not clear how much the two parameters if used together would complement each other towards determining stable communication topologies. The above questions form the motivation for the research conducted towards this paper and we seek to find answers to these questions. At moderate and high node velocity, given a choice between (i) operating the network with a larger pause time for the mobile nodes, but not letting any node to be permanently static or (ii) allowing certain fraction of the nodes to remain static all the time and letting the remaining nodes to move all the time, our hypothesis is that the first strategy would contribute towards a relatively more stable communication topology. This is because, we conjecture that though a certain percentage of the nodes are permanently static, the lifetime of the links is still heavily impacted by the percentage of mobile nodes and this will hold true unless the percentage of static nodes is at least 
significantly greater than the percentage of mobile nodes or the maximum node velocity is low. On the other hand, the network could be relatively more stable if all the mobile nodes pause for an appreciable time (especially when the maximum node velocity is high, but all the mobile nodes pause for a longer time), compared to letting only certain nodes to be static all the time and requiring the rest to move all the time. We also hypothesize that the stability of the Degree-based CDS would significantly increase with increase in the percentage of static nodes and/or the amount of pause time (compared to the CDS lifetime incurred when all nodes were mobile) as there are good chances that an appreciable fraction of the non-mobile nodes are also part of the Degree-based CDS and contribute towards its stability and cover the non-CDS nodes that could be mobile; on the other hand, the lifetime of the Maximum Stable CDS would only marginally increase, as it would be difficult to find a connected mobile graph that exists for a longer time even in the presence of a certain fraction of static nodes and/or nodes that have paused for awhile. The simulation results confirm all of our above hypotheses.

The rest of the paper is organized as follows: Section 2 presents a heuristic to determine Degreebased CDS and evaluates its run-time complexity. Section 3 presents the Maximum Stable CDS algorithm to determine a sequence of stable CDSs, explains its proof of correctness and illustrates its working with an example. Section 4 presents the simulation results evaluating the lifetime and node size of the Maximum Stable CDS vis-a-vis the Degree-based CDS under an extensive set of mobility scenarios varying the maximum node velocity, pause time and the percentage of static nodes for each level of node mobility. Section 5 differentiates our work from related work. Section 6 concludes the paper. Throughout the paper, the terms 'node' and 'vertex', 'link' and 'edge' are used interchangeably. They mean the same.

\section{HEURISTIC FOR DEGREE-BASED CONNECTED DOMINATING SET}

In this section, we first describe a heuristic that could be used to determine the CDS for a network graph based on the degree (the number of neighbors of a vertex) in the graph. We then illustrate examples to show the execution of the CDS construction heuristic. We finally describe an algorithm that can be used to validate the existence of a CDS at any time instant.

\subsection{Generic Heuristic to Determine a CDS}

The overall idea is to give preference (for inclusion to the CDS) for vertices that have a larger degree. As indicated in the pseudo code of Figure 1, we maintain three lists: CDS Node List vertices that are part of the CDS; Uncovered Node List - vertices that are yet to be covered by a node in the CDS Node List; Candidate Node List - vertices that are covered by a node in the CDS Node List, but not yet considered for inclusion to the CDS Node List. Initially, the CDS Node List is empty and the Uncovered Node List is the set of all the vertices in the graph; to start with, the Candidate Node List has a single entry corresponding to the vertex that has the largest degree. The Candidate Node List is implemented as a priority queue and the vertices in the Candidate Node List are stored in the decreasing order of their degree (the vertex with the largest degree is the first vertex to be removed from this list).

In each iteration, we remove a vertex from the Candidate Node List and if it has one or more uncovered neighbor nodes, then the vertex is added to the CDS Node List and the newly covered neighbor nodes are removed from the Uncovered Node List and included in the Candidate Node List. If a vertex removed from the Candidate Node List has no uncovered neighbors, then the vertex is not included in the CDS Node List. We repeat the above procedure until the Uncovered Node List gets empty or there are no more vertices in the Candidate Node List. If the Candidate Node List gets empty while the Uncovered Node List is not yet empty, then it implies the 
underlying graph is not connected (i.e., all the vertices are not in one component). If the underlying graph is connected, the iterations stop when there are no more vertices in the Uncovered Node List (this implies, all the vertices in the graph are covered by at least one node in the CDS Node List). Note that the degree score for a node is determined offline (prior to the execution of the heuristic) and is not updated during the execution of the heuristic. In other words, the ordering of the nodes in the Candidate Node List in each iteration is based on the initial (static) degree values input to the heuristic. Thus, for example, if two nodes $u$ and $v$ with degree scores of 4 and 6 respectively are in the Candidate Node List, but node $v$ has only two uncovered neighbors whereas node $u$ has three uncovered neighbors, node $v$ with a relatively larger degree score would still be ahead of node $u$ in the Candidate Node List.

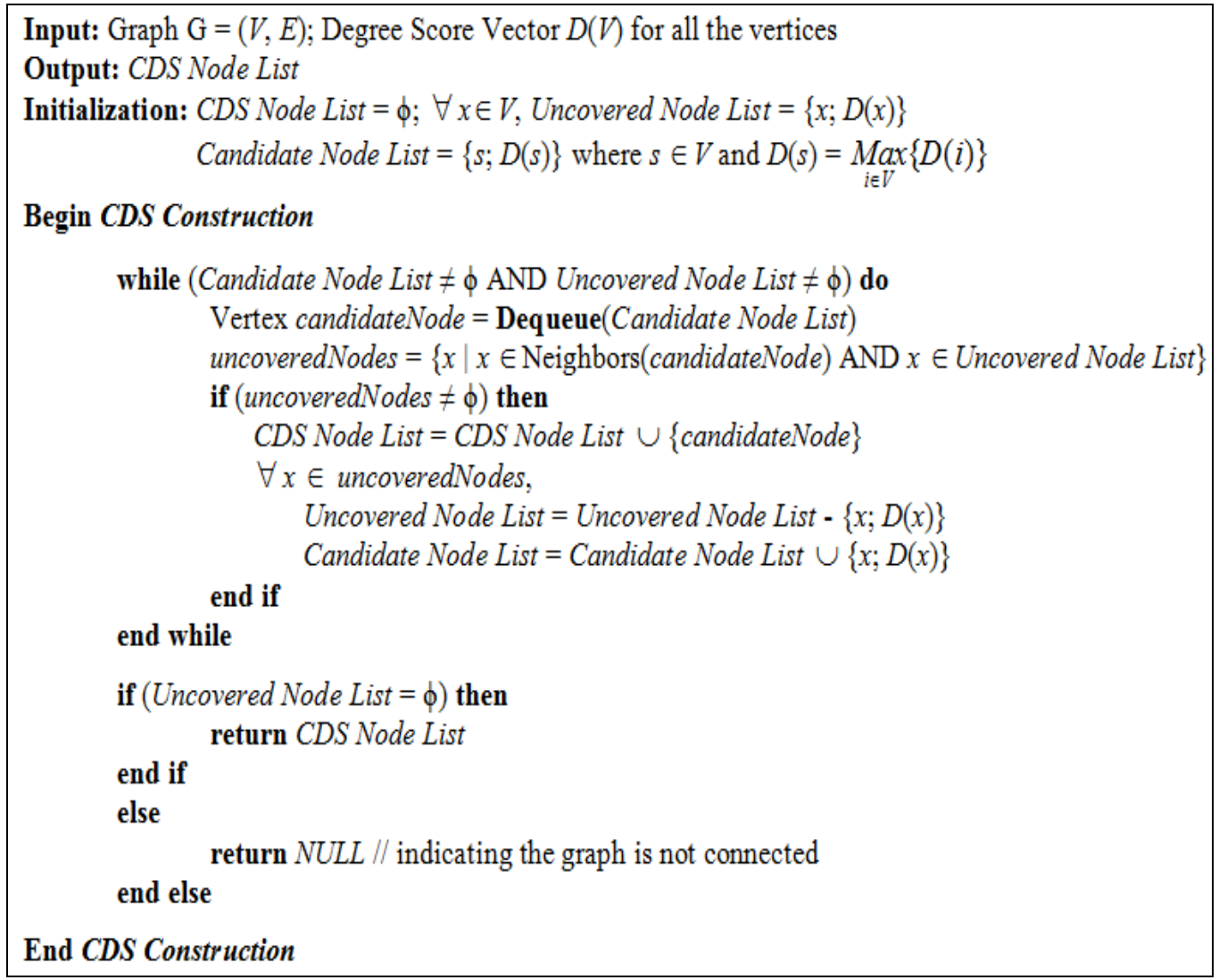

Figure 1. Pseudo Code for a CDS Construction Heuristic based on Node Degree

The time complexity for the CDS construction heuristic is $\mathrm{O}(V \log V+E \log V)$, where $V$ and $E$ are respectively the number of vertices and edges in the network graph; $\mathrm{O}(\log V)$ is the time complexity for a dequeue operation in a priority queue (Candidate Node List) maintained as a Max-Heap [8] as well as the time complexity to include a vertex in the Candidate Node List by visiting the neighbors of the vertex that is added to the CDS Node List. We could run the while loop at most $V$ times, once for each vertex, and across all such iterations, the entire set of edges are traversed and the end vertices of the edges are considered for inclusion to the Candidate Node List. Hence, the overall time complexity of the generic heuristic to construct a Degree-based CDS is $\mathrm{O}((V+E) \log V)$. 


\subsection{Example to Construct Degree-based CDS}

In this section, we show the execution of the degree heuristic on a sample network graph. For each vertex in Figure 2, the ID is indicated inside the circle and the degree of the vertex is indicated outside the circle. The last graph in each of these figures is a CDS graph comprising of only edges between two CDS nodes (indicated as solid lines) and edges between a non-CDS node and a CDS node (indicated as dotted lines).

Figure 2 illustrates the execution of the heuristic to determine Degree-based CDS: in iteration 3, even though vertices 3 and 7 have the same larger degree (3), vertex 3 is not considered for inclusion to the CDS because all of its three neighbors are already covered (i.e., none of the neighbors of vertex 3 are in the Uncovered Node List); on the other hand vertex 7 has one uncovered neighbor and is hence included to the CDS Node List. The sequence of vertices included in the Degree-based CDS are 2, 4, 7 and 6. One significant observation could be made in the toy example shown in Figure 2: Vertex 3 with a relatively higher degree (3) is connected to two high-degree vertices (vertices 2 and 7), but does not contribute much in terms of covering nodes that are yet in the Uncovered Nodes List once vertices 2 and 7 get into the CDS Node List. Thus, vertex 3 is a highly enclosed vertex that is not exposed much to vertices outside its own community. On the other hand, even if vertex 2 gets into the CDS Node List, vertex 7 could also get into the CDS Node List, because they are connected to two different vertices/communities (vertices 8 and 6) that only either of them could cover, but not both.
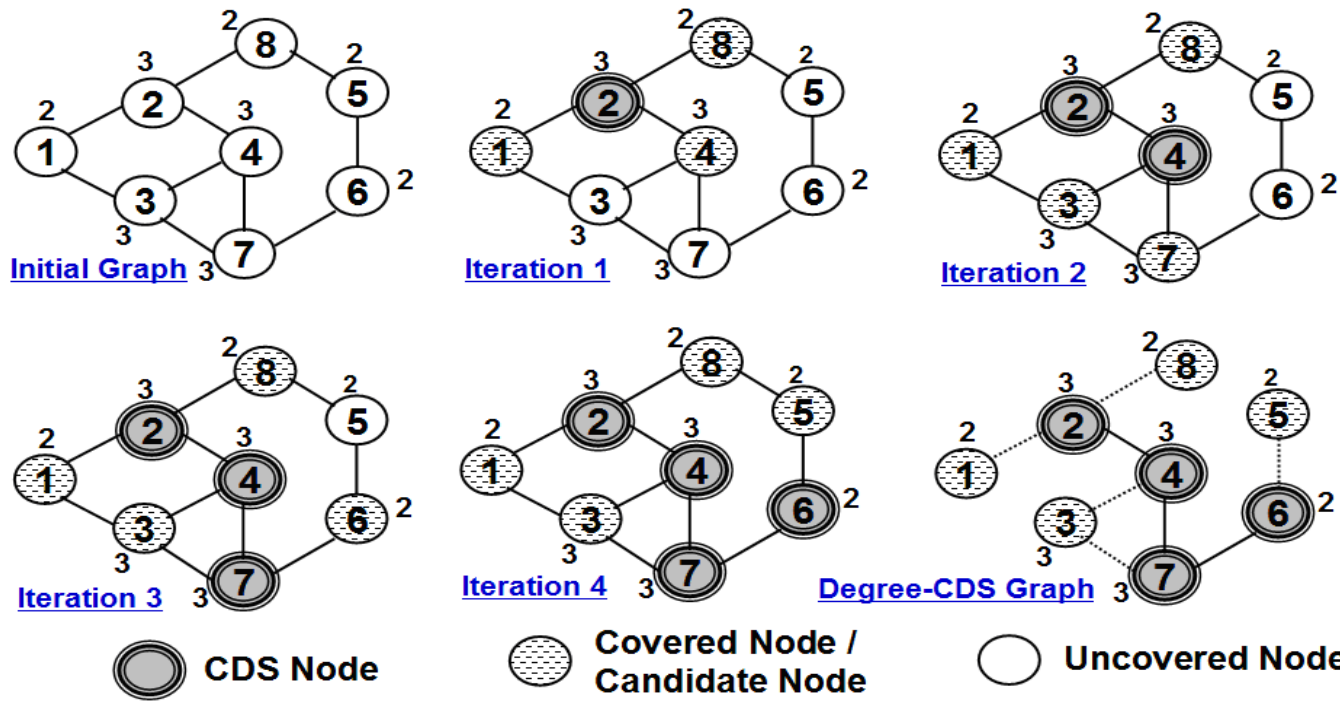

\section{Candidate Node}

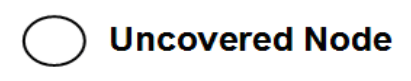

Figure 2. Example to Illustrate the Construction of a Degree-based CDS

\subsection{Algorithm to Validate the Existence of a CDS}

We now present the algorithm that we use to validate the existence of a CDS at any time instant. This algorithm would be very useful in discrete-event simulations when we tend to use a CDS that existed at an earlier time instant (say, $t$-1) at a later time instant $(t)$. That is, given a CDS Node List that was connected (i.e., the CDS nodes are reachable from one another directly or through one or more intermediate CDS nodes) and covered the non-CDS nodes in the network graph at time instant $t-1$, we want to validate whether the same holds true at time instant $t$. We do so by first constructing a CDS graph based on the CDS Node List at time instant $t$. All the vertices in the network are part of the CDS graph and the edges in the CDS graph are those that 
exist between any two CDS nodes as well as between a CDS node and a non-CDS node at time instant $t$. We check if the CDS Node List is connected at time instant $t$ by running the Breadth First Search (BFS) [8] algorithm, starting from an arbitrarily chosen CDS node and see if every other CDS node could be reached as part of the BFS traversal only on the CDS-CDS edges. If all the CDS nodes could be visited, the CDS Node List is considered to be connected. We then check whether every non-CDS node has an edge to at least one CDS node at time $t$. If both the validations (connectivity of the CDS Node List and coverage of every non-CDS node by at least one CDS node) are true, then we consider the CDS to exist at time instant $t$.

\section{BENCHMARKING ALGORITHM FOR MAXIMUM STABILITY CONNECTED DOMINATING SET}

In this section, we describe a benchmarking algorithm [9] proposed to determine a sequence of longest-living connected dominating sets for MANETs such that the number of transitions is the global minimum and the average lifetime of the CDS is the maximum. We refer to the algorithm as the Maximum Stable CDS algorithm; it works on the basis of graph intersections and assumes the availability of information about topology changes for the entire duration of the network session. The algorithm introduces the notion of a mobile graph $\mathrm{G}(t, \ldots ., t+k)=\mathrm{G}(t) \cap \mathrm{G}(t+1)$ $\cap \mathrm{G}(t+2) \cap \ldots \cap \mathrm{G}(t+k)$, wherein $\mathrm{G}(t), \mathrm{G}(t+1), \mathrm{G}(t+2), \ldots, \mathrm{G}(t+k)$ are static graphs (snapshots) of the network at time instants $t, t+1, t+2, \ldots, t+k$ respectively. A mobile graph $\mathrm{G}(t, \ldots ., t+k)$ is thus a graph that essentially captures the vertices and edges that exist in the network for all the time instants $t, t+1, t+2, \ldots, t+k$. The Maximum Stable CDS algorithm works as follows: Whenever a CDS is required at time instant $t$, we determine a mobile graph $\mathrm{G}(t, \ldots ., t+k)$ such that $\mathrm{G}(t, \ldots ., t+k)$ is connected and $\mathrm{G}(t, \ldots, t+k+1)$ is not connected. We determine a CDS on such a longest-living connected mobile graph $\mathrm{G}(t, \ldots ., t+k)$ and repeat the above procedure for the duration of the network session to obtain a sequence of longest-living CDSs such that the number of transitions needed to change from one CDS to another is the optimum (minimum). We say the optimum number of transitions incurred is the global minimum because the algorithm assumes the knowledge of the entire topology changes and always chooses the longest-living mobile graph since the time instant starting from which a CDS is needed. Later, we also prove that the number of transitions accomplished by any other CDS construction algorithm cannot be below the value obtained for the Maximum Stable CDS algorithm. Accordingly, the average of the lifetimes of the longest-living connected dominating sets in the sequence determined by the Maximum Stable CDS algorithm for the duration of the network session is the global maximum and can serve as a benchmark to compare the average lifetime incurred with any other sequence of connected dominating sets (like the Degree-based CDSs) under identical conditions. 


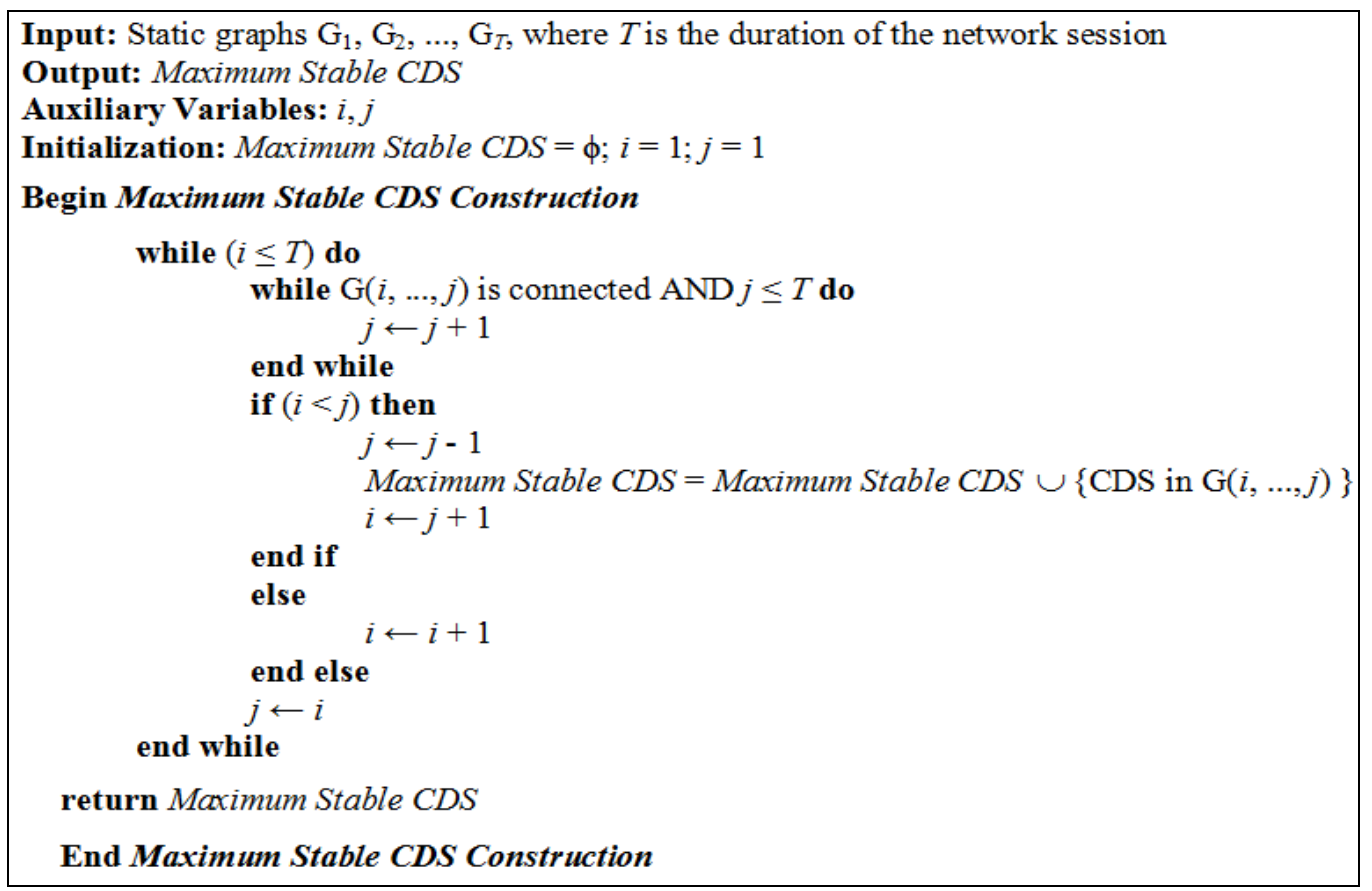

Figure 3. Pseudo Code for the Benchmarking Algorithm to Determine Maximum Stable CDS

As seen in the pseudo code for the Maximum Stable CDS algorithm (Figure 3), it does not matter what CDS construction heuristic we use to determine a CDS in each of the longest-living mobile graphs $\mathrm{G}(i, \ldots, j)$, because the CDS would not exist in the mobile graph $\mathrm{G}(i, \ldots, j+1)$ as the latter would not be a connected graph. In this research, we determine the connected dominating sets in the longest-living mobile graph $\mathrm{G}(i, \ldots, j)$ based on the degree of the vertices in the mobile graph $\mathrm{G}(i, \ldots, j)$. The average lifetime of the Maximum Stable CDS would not be affected because of this; however, the size of the Maximum Stable CDS is likely to be affected. As Degree-based CDSs are likely to incur a lower CDS Node Size, it would be only appropriate to determine the connected dominating sets in each of the longest-living mobile graphs $\mathrm{G}(i, \ldots, j)$ using the degree of the vertices in the mobile graph so that the Maximum Stable CDS would incur a lower CDS Node Size and at the same time incur the maximum lifetime. The time complexity of the Maximum Stable CDS algorithm depends on the time complexity of the underlying heuristic used to determine the CDSs in each of the mobile graphs. As one can see in the pseudo code (Figure 3 ), there could be at most $T$ mobile graphs (where $T$ is the number of static graph snapshots, number of graph samples) on which a CDS heuristic needs to be run; if we use a $\Theta\left(V^{2}\right)$ heuristic to determine Degree-based CDSs, then the overall time complexity of the Maximum Stable CDS algorithm is $\mathrm{O}\left(V^{2} T\right)$.

\subsection{Proof of Correctness}

We now present an informal proof of correctness of the Maximum Stable CDS algorithm. For a more formal proof, the interested reader is referred to [9]. To prove that the Maximum Stable CDS algorithm finds a sequence of long-living CDSs that incur the minimum number of transitions (say $m$ ), assume the contrary - i.e., there exists a hypothetical algorithm that determines a sequence of CDSs that undergo $n$ transitions such that $n<m$. We will now explore whether this is possible. For $n<m$ to exist, there has to be an epoch of time instants $[p, \ldots, s]$ that is a superset of an epoch of time instants $[q, . ., r]$ such that $p<q<r<s$ and that the hypothetical algorithm was able to find a CDS that existed during time instants $[p, \ldots, s]$, but the Maximum 
Stable CDS algorithm can only find a CDS that existed during time instants $[q, \ldots, r]$ and had to go a transition at time instant $r+1$. This implies the Maximum Stable CDS algorithm could not find a connected mobile graph $\mathrm{G}(q, \ldots, r+1)$ and could only find a connected mobile graph $\mathrm{G}(q$, $\ldots, r)$. This means there existed no connected mobile graph from time instants $[q, \ldots, s]$ and hence there is no connected mobile graph from time instants $[p, \ldots, s]$. If that is the case, it would not be possible to find a CDS that exists from time instants $[p, \ldots, s]$; thus, the sequence of connected dominating sets determined by the hypothetical algorithm has to undergo at least as many transitions as those determined by the Maximum Stable CDS algorithm, which is a contradiction to our initial assumption that $n<m$. Hence, the number of transitions $(m)$ incurred by the sequence of long-living CDSs determined by the Maximum Stable CDS algorithm serves as a lower bound for the number of transitions $(n)$ incurred by any other algorithm/heuristic to determine a sequence of CDSs.

\subsection{Example}

Figure 4 presents an example to illustrate the execution of the Maximum Stable CDS algorithm on a sequence of five static graphs $G_{1}, \ldots, G_{5}$ and a comparison of the number of transitions (changes from one CDS to another) and CDS Node Size incurred for Maximum Stable CDS visa-vis a Degree-based CDS on the same sequence of static graphs. We see that there exists a connected mobile graph $\mathrm{G}(1,2,3)$ and a connected mobile graph $\mathrm{G}(4,5)$. Hence, it would suffice to use one CDS determined on the mobile graph $\mathrm{G}(1,2,3)$ and another CDS determined on the mobile graph $G(4,5)$ - resulting in only one CDS transition across the five static graphs. On the other hand, the Degree-based CDS determined on an individual static graph does not exist in the subsequent static graph. Hence, we end up determining a new Degree-based CDS on each of the five static graphs - resulting in a total of four CDS transitions across the five static graphs. In Figure 4, we use a degree-based heuristic to determine a CDS on the two mobile graphs.

We do observe a tradeoff between CDS Node Size and the number of CDS transitions. The average CDS Node Size incurred for the Maximum Stable CDS is $(3 * 5+2 * 7) / 5=5.8$, whereas the average CDS Node Size incurred for Degree-based CDS is $(3 * 4+2 * 5) / 5=4.4$. The mobile graphs $\mathrm{G}(1,2,3)$ and $\mathrm{G}(4,5)$ have relatively fewer links than the individual static graphs. Hence, it is not possible to cover a larger number of nodes with the inclusion of few nodes in the CDS. As a result, the Maximum Stable CDS incurs a relatively larger CDS Node Size compared to that of the Degree-based CDS.

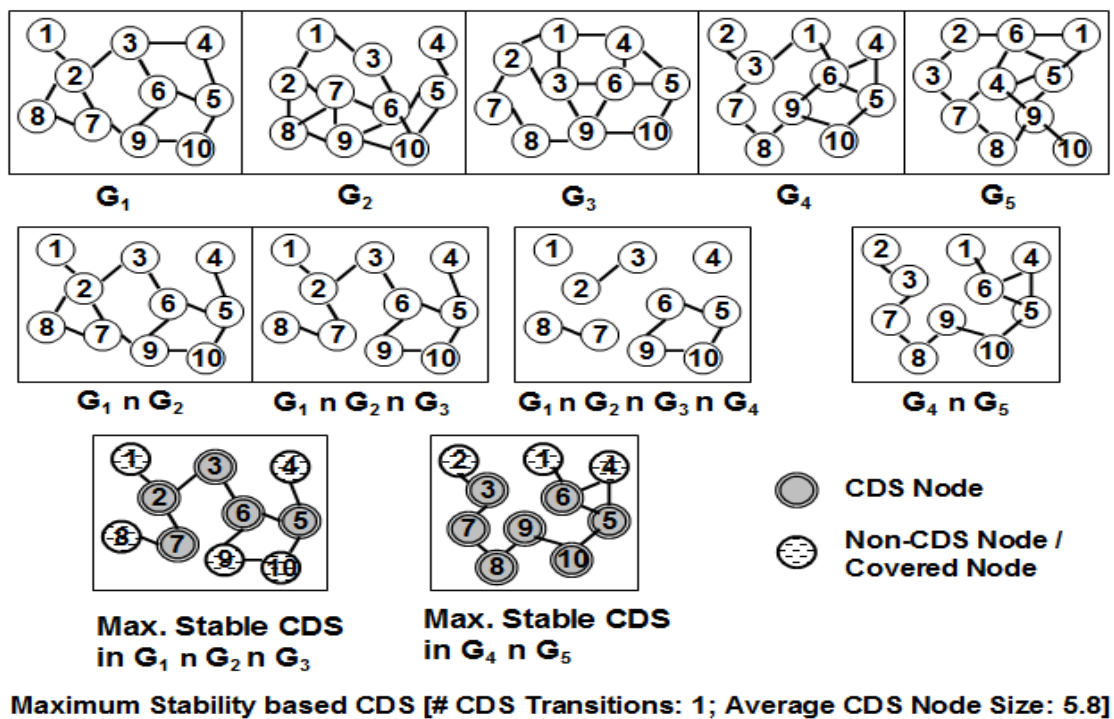




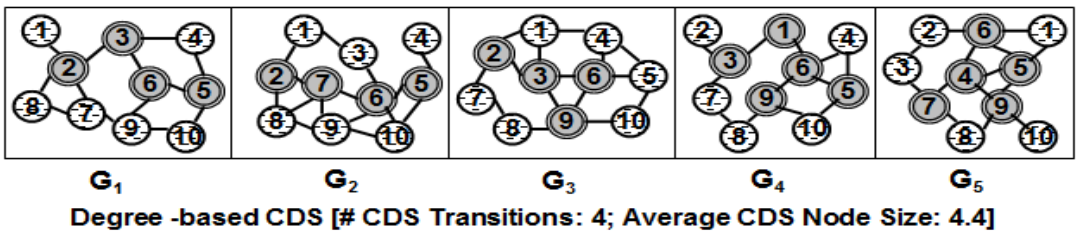

Figure 4. Example to Illustrate the Execution of the Maximum Stable CDS Benchmarking Algorithm and a Comparison of Maximum Stable CDS vs. Degree-based CDS

\section{SIMULATIONS}

We conducted the simulations in a discrete-event simulator implemented in Java. We assume a network of dimensions $1000 \mathrm{~m} \times 1000 \mathrm{~m}$; the number of nodes is fixed as 100 and the transmission range of the nodes is fixed at $250 \mathrm{~m}$; for these values, on average, there are $\pi * 250 * 250 * 100 /(1000 * 1000)=20$ neighbors per node. The overall connectivity of the network for the above conditions is observed to be more than $99.5 \%$. Initially, the nodes are uniformrandomly distributed throughout the network. The number of static nodes in the network is varied with values of 0,20 and 40 . Since the total number of nodes in the network is 100 , the above values for the number of static nodes also represent the percentage of static nodes in the network. A node designated to be static in the beginning of the simulation does not move at all for the duration of the simulation.

The node mobility model used is the Random Waypoint Model [10], wherein the maximum velocity of each mobile node is $v_{\max }$ and it is varied with values of $5 \mathrm{~m} / \mathrm{s}$ (low mobility), $25 \mathrm{~m} / \mathrm{s}$ (moderate mobility) and $50 \mathrm{~m} / \mathrm{s}$ (high mobility). The maximum pause time (ptime $e_{\max }$ ) for a mobile node is varied from 0 to 400 seconds, in increments of 50 seconds. According to the Random Waypoint Model, each mobile node starts from its initial location and chooses to move to an arbitrary location (located within the network boundaries) with a velocity that is uniformrandomly selected from the range $\left[0, \ldots, v_{\max }\right]$; after moving to the chosen location, the node pauses for a time uniform-randomly selected from the range $\left[0, \ldots\right.$, ptime $\left._{\max }\right]$ and stays in that location for the selected pause time; the node then chooses another arbitrary location (within the network) and moves to the chosen location with a new velocity that is uniform-randomly chosen from the range $\left[0, \ldots, v_{\max }\right]$ and pauses there for awhile for a different pause time chosen uniformrandomly from the range $\left[0, \ldots\right.$, ptime $\left._{\max }\right]$. In other words, a node moves in a straight line from one location to another chosen location with a particular velocity and after reaching the targeted location, the node pauses there for awhile and then chooses another target location to move with a velocity that could be different from the one used before and pauses there for awhile that could be different from the previous pause time. The velocity and pause time for a node during each of its movements are intended to be different and independent of the past. A mobile node moves like this for the duration of the simulation session and generates a mobility profile for a particular combination of values for the maximum node velocity and maximum pause time. The collection of the mobility profiles of all the nodes is stored in a mobility profile file and is feed into the heuristic/algorithm for determining a Degree-based CDS and the Maximum Stable CDS. We generate 100 such mobility profile files for each of the combinations of the values for the number of static nodes $(0,20$ and 40), the maximum node velocity $(5 \mathrm{~m} / \mathrm{s}, 25 \mathrm{~m} / \mathrm{s}$ and $50 \mathrm{~m} / \mathrm{s})$ and maximum pause time $(0,50,100,150,200,250,300,350$ and 400 seconds). Hence, we have a total of $3 * 3 * 9=81$ scenarios. The decision of whether a node will be permanently static or mobile is made prior to creating each of the mobility profile files. 
We run the simulations for each mobility profile file and average the results observed for the CDS Lifetime and CDS Node Size for the Degree-based CDS as well as the Maximum Stable CDS. We start the simulations at time $0 \mathrm{sec}$ (based on the initial distribution of the nodes in a particular mobility profile file) and run the simulations for a period of 1000 seconds, sampling the network for every 0.25 seconds of the simulation. We take a snapshot of the network at each of these sampling time instants (referred to as the static graphs). We use the following approach to run the simulations for the degree-CDS: Whenever a CDS is needed at a particular time instant $t$, we determine the degree scores of the nodes based on the static graph snapshot of the network at time instant $t$ and feed in these scores to the CDS construction heuristic (described in Section 2.1). The CDS determined at time instant $t$ is used for the subsequent sampling time instants as long as it exists (validated using the algorithm presented in Section 2.3). When the currently known CDS is observed to no longer exist at a particular time instant, we repeat the above procedure. We continue like this for the duration of the simulation and measure the lifetime and node size for the sequence of degree-based connected dominating sets used for the particular mobility profile file. We average the results for these two metrics (Figures 8-16) observed for all the mobility profile files generated for a particular combination of values for the number of static nodes and $v_{\max }$. In the case of the Maximum Stable CDS algorithm, for a particular simulation run under a mobility profile file, we determine a sequence of connected mobile graphs for the duration of the simulation session and run the Degree-based CDS construction heuristic to determine a sequence of connected dominating sets. We repeat this procedure for all the mobility profile files generated and determine the average of the lifetime and node size for the sequence of Maximum Stable CDSs for the particular combination of values for the number of static nodes, $v_{\max }$ and ptime $_{\max }$.

\subsection{Fraction of Static Nodes at any Time}

For each combination of the simulation conditions (i.e., the 81 scenarios listed above), we examined the average fraction of static nodes in the network at any time instant during the simulation time period of 1000 seconds. In this pursuit, we determined the fraction of nodes that are static at each of the sampling time instants (i.e., nodes that do not move; this includes the nodes that are permanently static and nodes that have paused for that time instant) and averaged this value across all the time instants, and further averaged over all the 100 mobility profile files for each scenario. For each scenario and across all the mobility profile files for the scenario, we do not observe any significant variation in the fraction of static nodes across the time instants.

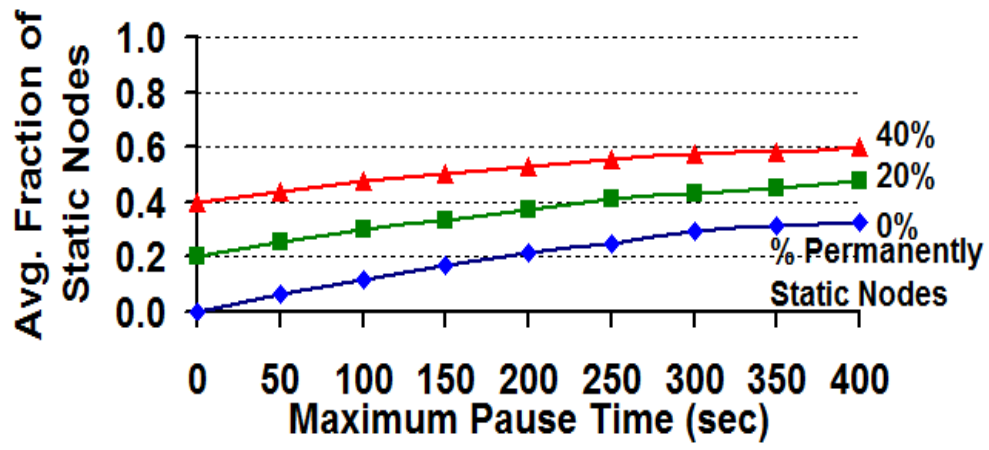

Figure 5. Average of Static Nodes at Low Node Mobility Scenarios $\left(v_{\max }=5 \mathrm{~m} / \mathrm{s}\right)$ 


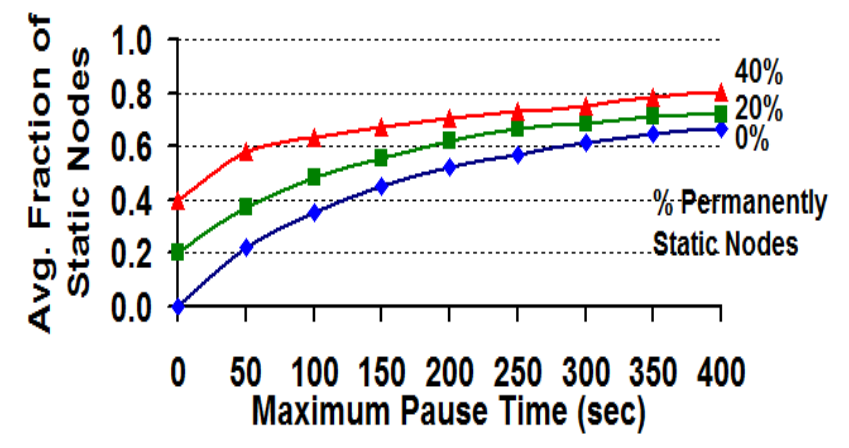

Figure 6. Average of Static Nodes at Moderate Node Mobility Scenarios ( $v_{\max }=25 \mathrm{~m} / \mathrm{s}$ )

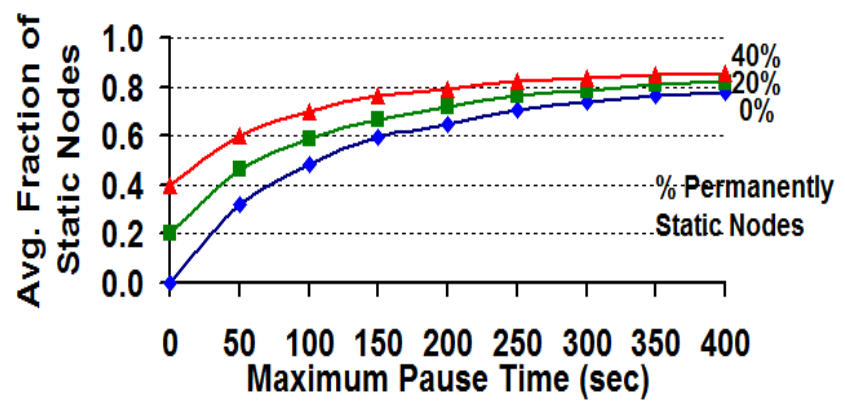

Figure 7. Average of Static Nodes at High Node Mobility Scenarios $\left(v_{\max }=50 \mathrm{~m} / \mathrm{s}\right)$

Figures 5-7 illustrate the average fraction of static nodes at any time instant in the network for $v_{\max }$ values of low, moderate and high node mobility $(5 \mathrm{~m} / \mathrm{s}, 25 \mathrm{~m} / \mathrm{s}$ and $50 \mathrm{~m} / \mathrm{s}$ respectively). Overall, we observe that for a given level of node mobility and the \% of permanently static nodes, as the pause time increases, the average fraction of static nodes increases. The nature of increase exhibits a concave down pattern (i.e., even though there is an increase with increase in pause time, the slope of the curve decreases with increase in pause time), especially at scenarios of moderate and high node mobility (see Figures 6-7). As we can see for scenarios of high node mobility (Figure 7), the fraction of static nodes reaches a plateau for a maximum pause time of 300 seconds, indicating that increasing the pause time beyond this value will not appreciably increase the fraction of static nodes in the network. On the other hand, for low mobility scenarios (Figure 5), the fraction of static nodes exhibits almost a linear increase with increase in the pause time (albeit, with a reduced slope as the $\%$ of permanently static nodes increases). Of course, when the $\%$ of permanently static nodes and pause time are considered together, we see that larger the values for each of these two parameters, the larger the fraction of static nodes in the network (i.e., the larger the chances of finding stable network-wide communication topologies).

We make two significant observations: (i) With regards to the nature of increase in the average fraction of static nodes is that at low node mobility scenarios $\left(v_{\max }=5 \mathrm{~m} / \mathrm{s}\right.$; Figure 5$)$, the magnitude of increase in the fraction of static nodes (as we increase the pause time from 0 seconds to 400 seconds) is relatively low (the increase is at most by a value of 0.32 when the $\%$ of permanently static nodes is $0 \%$ and is at most 0.20 when the $\%$ of permanently static nodes is $40 \%)$; on the other hand, for moderate and high node mobility scenarios $\left(v_{\max }=25\right.$ and $50 \mathrm{~m} / \mathrm{s}$; Figures 6-7), the magnitude of increase in the fraction of static nodes as we increase the pause time from 0 seconds to 400 seconds is appreciably high (the increase is by values of $0.67-0.78$ when the $\%$ of permanently static nodes is $0 \%$ and is by values of $0.40-0.46$ when the $\%$ of permanently static nodes is $40 \%$ ). (ii) For a given level of node mobility, though the rate of increase in the fraction of static nodes decreases with increase in the $\%$ of permanently static 
nodes, we could observe two different trends at moderate/high node mobility scenarios vis-a-vis low node mobility scenarios. At moderate and high node mobility scenarios (Figures 6-7), the curves for the fraction of the static nodes for different values of the \% of permanently static nodes converge towards each other. On the other hand, for low node mobility scenarios (Figure 5), the curves for the fraction of the static nodes for different values of the \% of permanently static nodes do not converge and continue to be distinctly different (higher for larger values of the $\%$ of permanently static nodes).

The above two observations help us to infer about the relative contribution of the $\%$ of static nodes and pause time towards the stability of network-wide communication topologies such as the CDS. We could now infer that between the two parameters, the pause time dominates in networks of moderate and high node mobility and is likely to contribute relatively more for the stability of network-wide communication topologies; whereas the \% of permanently static nodes dominates in networks of low node mobility and is likely to contribute relatively more for the stability of network-wide communication topologies.

\subsection{CDS Lifetime and CDS Node Size}

As expected, the Maximum Stable CDS incurred a larger CDS lifetime compared to that of the Degree-based CDS and at the same time the difference in the CDS Node Size is significantly low (sometimes, the Maximum Stable CDS incurs a Node Size that is smaller than that of the Degreebased CDS). Under conditions of moderate and high node mobility $\left(v_{\max }=25\right.$ and $50 \mathrm{~m} / \mathrm{s}$; see Figures 11-16), for a given \% of static nodes, the difference in the CDS lifetimes between the Maximum Stable CDS and Degree-based CDS significantly decreases with increase in the pause time. This could be attributed to the increase in the average fraction of static nodes in the underlying network topology and there is a good chance of accommodating a larger of number of static nodes in the connected dominating sets under both Degree-based CDS and Maximum Stable CDS. Thus, for moderate and high node mobility scenarios, the magnitude of reduction in the difference in the CDS lifetimes (Maximum Stable CDS vs. Degree-based CDS) is much more prominent (as we increase the pause time for a fixed number of static nodes and $v_{\max }$ ) compared to the difference in CDS lifetimes observed when we increase the $\%$ of static nodes for a fixed value of pause time and $v_{\max }$.

For scenarios of moderate and high maximum node velocity (see Figures 11-16), if we operate at a larger node pause time for the mobile nodes (and have $0 \%$ or more permanently static nodes), it would lead to much more stable communication topologies than letting a certain fraction of the nodes to be permanently static, but requiring the mobile nodes to move all the time. When we simultaneously operate at larger values of $v_{\max }$ and larger values of ptime $e_{\max }$, we observe larger pause times to be very effective in increasing the stability of the CDSs and the lifetime of the Degree-based CDS approaches the lifetime of the Maximum Stable CDS, irrespective of the \% of permanently static nodes (with increase in the pause time, the ratio of the Maximum Stable CDS Lifetime and Degree-based CDS Lifetime approaches 1 for all values of the $\%$ of permanently static nodes, as observed in Figures 18-19). This vindicates our hypothesis that for networks of moderate and high node mobility, the pause time of the mobile nodes has a relatively better impact in maximizing the stability of the communication topologies rather than the $\%$ of static nodes. 

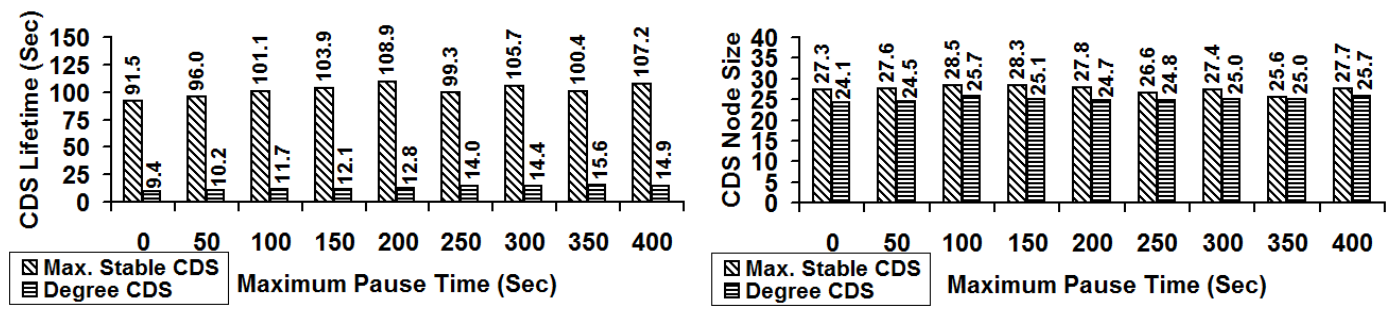

Figure 8. Maximum Stable CDS vs. Degree CDS $\left[v_{\max }=5 \mathrm{~m} / \mathrm{s} ; 0 \%\right.$ static nodes $]$
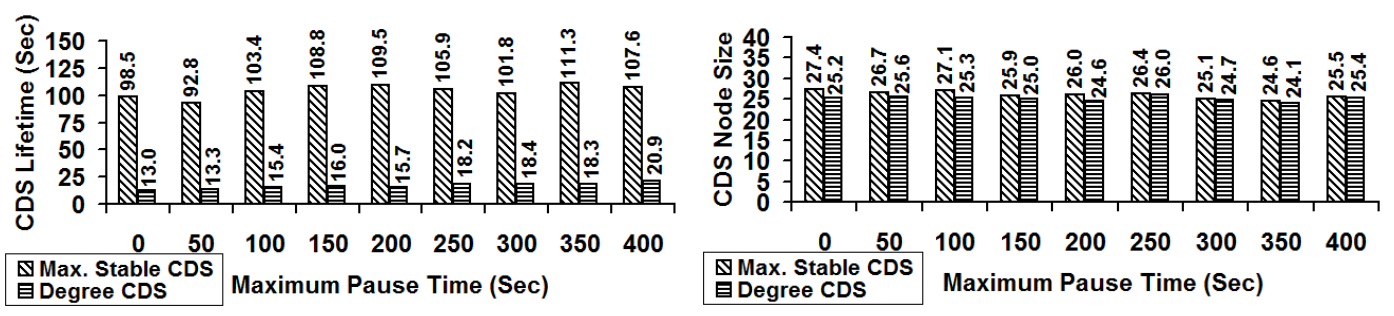

Figure 9. Maximum Stable CDS vs. Degree CDS $\left[v_{\max }=5 \mathrm{~m} / \mathrm{s} ; 20 \%\right.$ static nodes]
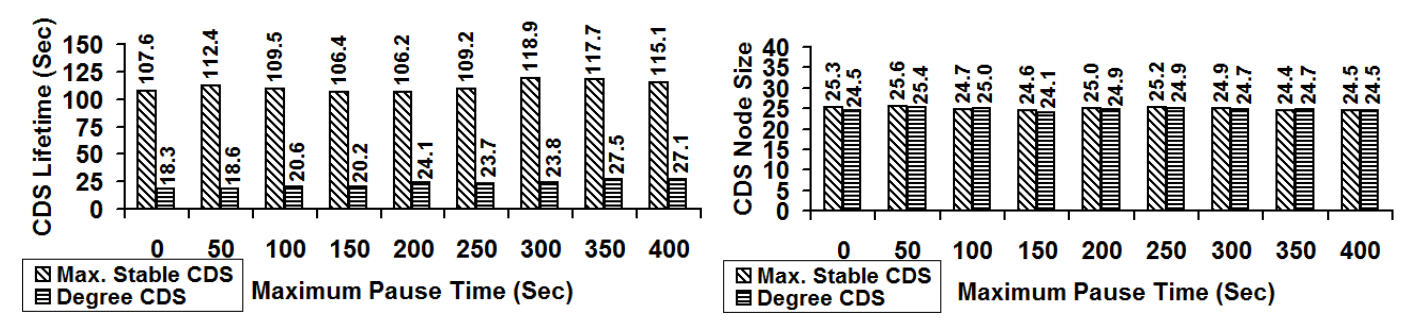

Figure 10. Maximum Stable CDS vs. Degree CDS [ $v_{\max }=5 \mathrm{~m} / \mathrm{s} ; 40 \%$ static nodes $]$
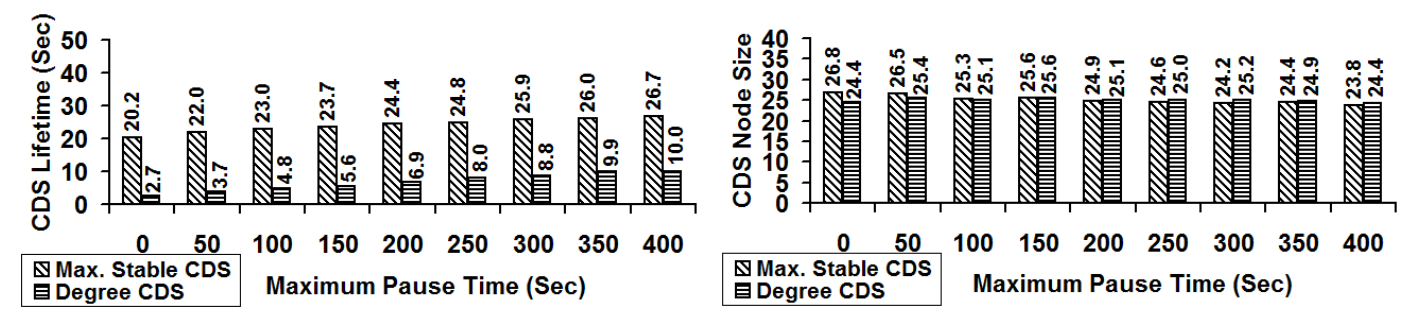

Figure 11. Maximum Stable CDS vs. Degree CDS $\left[v_{\max }=25 \mathrm{~m} / \mathrm{s} ; 0 \%\right.$ static nodes $]$

For scenarios of low node mobility (see Figures 8-10), as a further vindication of our hypothesis and the observation made in Section 4.1, the connected dominating sets determined in networks with a larger percentage of static nodes (and even a zero pause time for the mobile nodes) are relatively more stable compared to that of the connected dominating sets determined in networks with larger value of pause time for the mobile nodes (but no static nodes). For scenarios of low node mobility, we observe the magnitude of reduction in the difference in the CDS lifetimes is relatively lower when we increase the maximum pause time $\left(\right.$ ptime $_{\max }$ ) for a given value of $v_{\max }$ and the $\%$ of static nodes in the network. For a given $\%$ of permanently static nodes, there is always a significantly large difference between the Maximum CDS Lifetime and Degree-based CDS Lifetime (the ratio of the lifetimes is much larger than 1, as observed in Figure 17). For a given $\%$ of permanently static nodes, as we increase the pause time, we also observe from Figure 17 that the decrease in the lifetime ratio is not much prominent compared to the decrease in the lifetime ratios in Figures 18-19. 

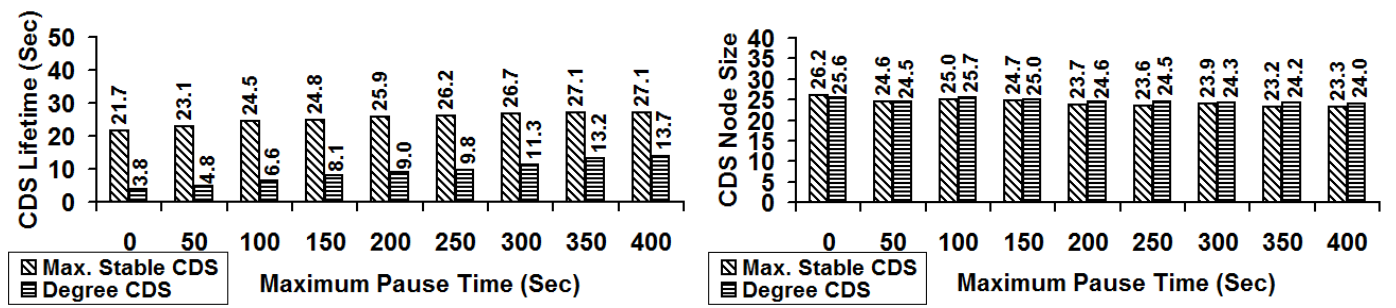

Figure 12. Maximum Stable CDS vs. Degree CDS [ $v_{\max }=25 \mathrm{~m} / \mathrm{s} ; 20 \%$ static nodes]
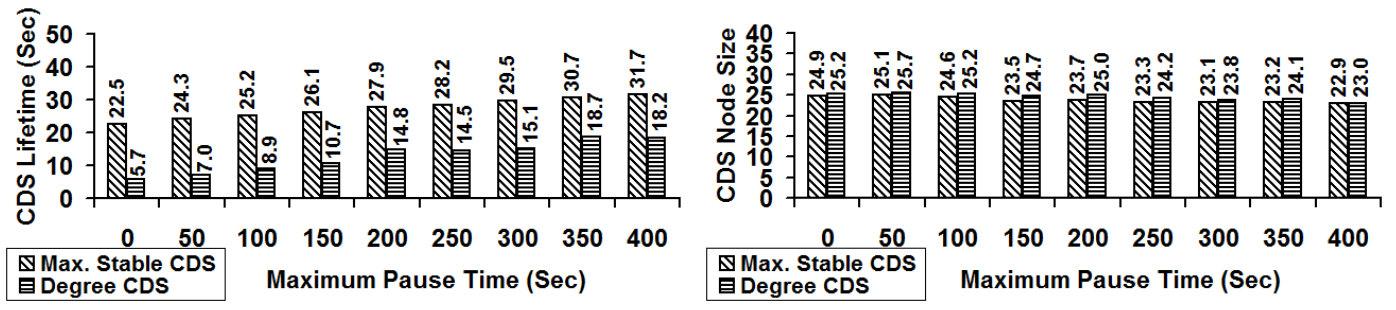

Figure 13. Maximum Stable CDS vs. Degree CDS [ $v_{\max }=25 \mathrm{~m} / \mathrm{s} ; 40 \%$ static nodes]
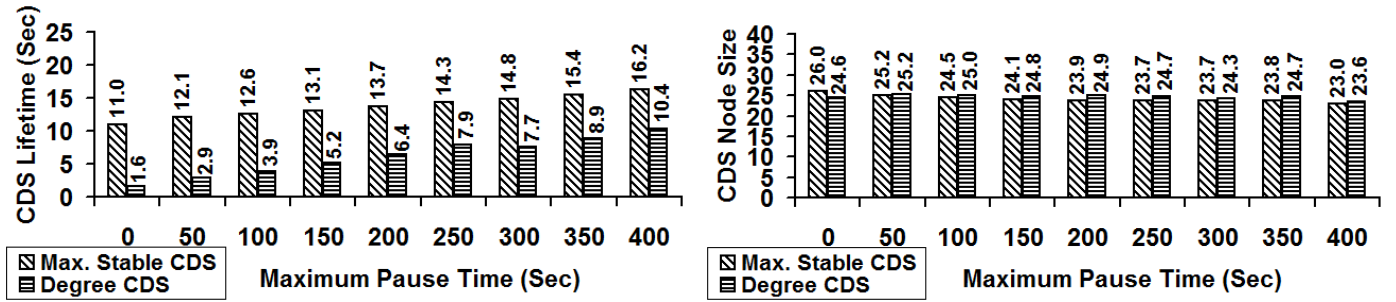

Figure 14. Maximum Stable CDS vs. Degree CDS $\left[v_{\max }=50 \mathrm{~m} / \mathrm{s} ; 0 \%\right.$ static nodes]
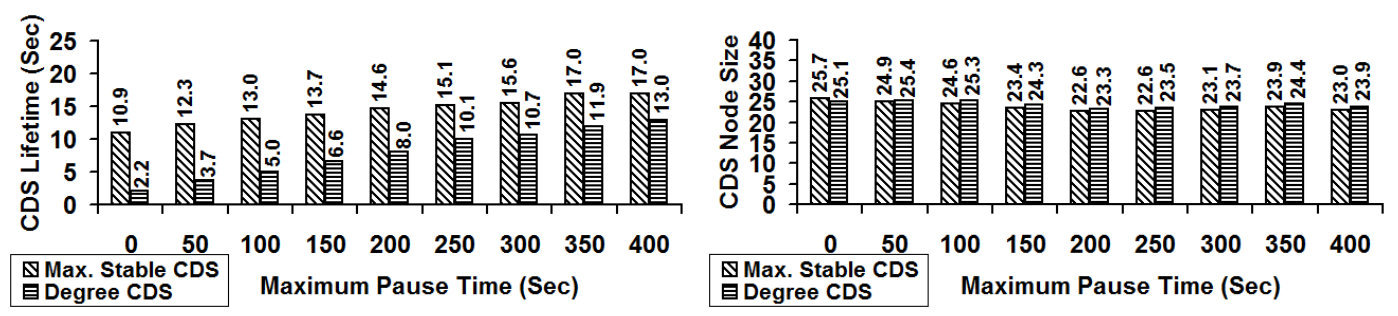

Figure 15. Maximum Stable CDS vs. Degree CDS [ $v_{\max }=50 \mathrm{~m} / \mathrm{s} ; 20 \%$ static nodes]

With regards to the CDS Node Size, we observe both the Maximum Stable CDS and Degreebased CDS to incur a CDS Node Size that is very much comparable; the Node Size for the Maximum Stable CDS is distinctly larger than that of the Degree-based CDS in low node mobility scenarios $\left(v_{\max }=5 \mathrm{~m} / \mathrm{s}\right)$ and the difference could be at most $15 \%$ (the difference decreases with increase in the $\%$ of permanently static nodes and is almost independent of the pause time). On the other hand, for moderate and high node mobility scenarios, the Maximum Stable CDS incurs only a slightly larger Node Size (at most 5\% larger) than the Degree-based CDS for lower values of maximum pause time; whereas as the maximum pause time increases, the difference in the Node Size narrows down and the Maximum Stable CDS starts incurring even a lower Node Size compared to the Degree-based CDS (at most 5\% lower). Overall, we could say that there is no appreciable difference in the Node Size for the Maximum Stable CDS and Degree-based CDS; hence, we do not see any stability-node size tradeoff for connected dominating sets, unlike the stability-related tradeoffs observed for unicast [13-14] and multicast communication [15] in MANETs. 

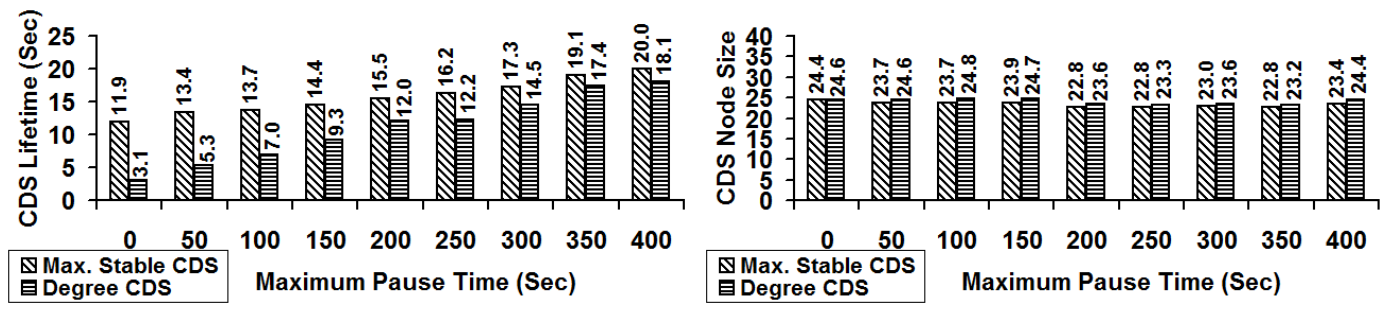

Figure 16. Maximum Stable CDS vs. Degree CDS [ $v_{\max }=50 \mathrm{~m} / \mathrm{s} ; 40 \%$ static nodes]
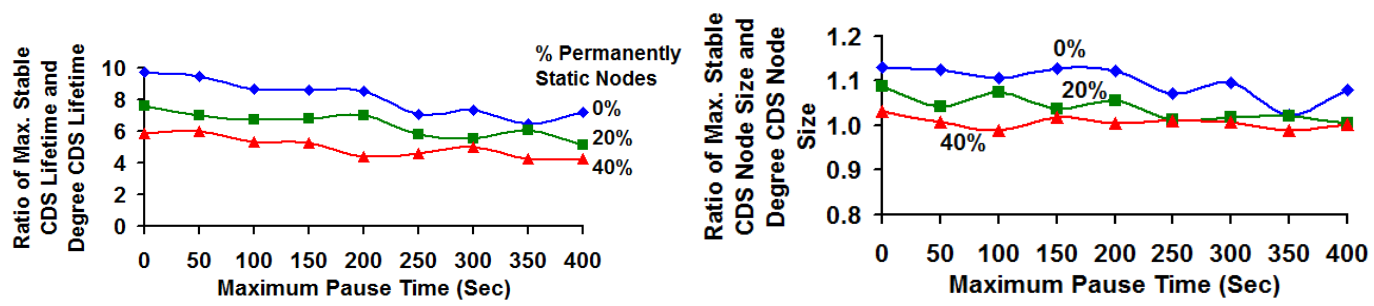

Figure 17. Performance Ratio of Maximum Stable CDS and Degree-based CDS $\left[v_{\max }=5 \mathrm{~m} / \mathrm{s}\right.$ ]
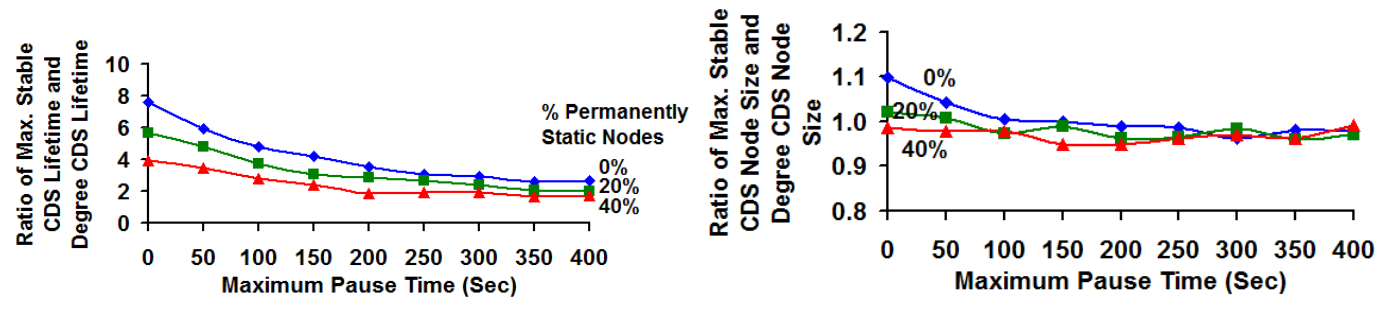

Figure 18. Performance Ratio of Maximum Stable CDS and Degree-based CDS [ $v_{\max }=25 \mathrm{~m} / \mathrm{s}$ ]
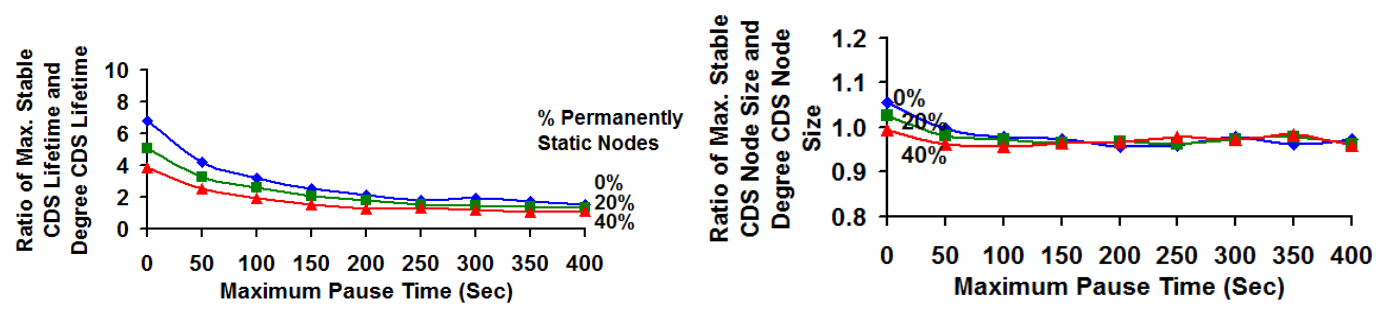

Figure 19. Performance Ratio of Maximum Stable CDS and Degree-based CDS $\left[v_{\max }=50 \mathrm{~m} / \mathrm{s}\right.$ ]

\subsection{Variation in the CDS Lifetime and CDS Node Size}

We find the maximum values for the average CDS Lifetime and average CDS Node Size with the Maximum Stable CDS as well as the Degree-based CDS across all the simulation scenarios. Using these maximum values, we compute the ratio of the average Maximum CDS Lifetime to the maximum of the Maximum Stable CDS Lifetime as well as the ratio of the average Degreebased CDS Lifetime to the maximum of the Degree-based CDS Lifetime for each of the simulation scenarios. Similarly, we compute the ratios of the average Maximum Stable CDS Node Size to the maximum of the Maximum Stable CDS Node Size as well as the ratios of the average Degree-based CDS Node Size to the maximum of the Degree-based CDS Node Size for each of the simulation scenarios. We then plot the data points for the Maximum Stable CDS Lifetime ratio vs. the Degree-based CDS Lifetime ratio as well as the data points for the 
Maximum Stable CDS Node Size vs. the Degree-based CDS Node Size. These plots (Figures 2022) could be used to infer the relative variations in the CDS Lifetime and CDS Node Size with the Maximum Stable CDS and the Degree-based CDS.
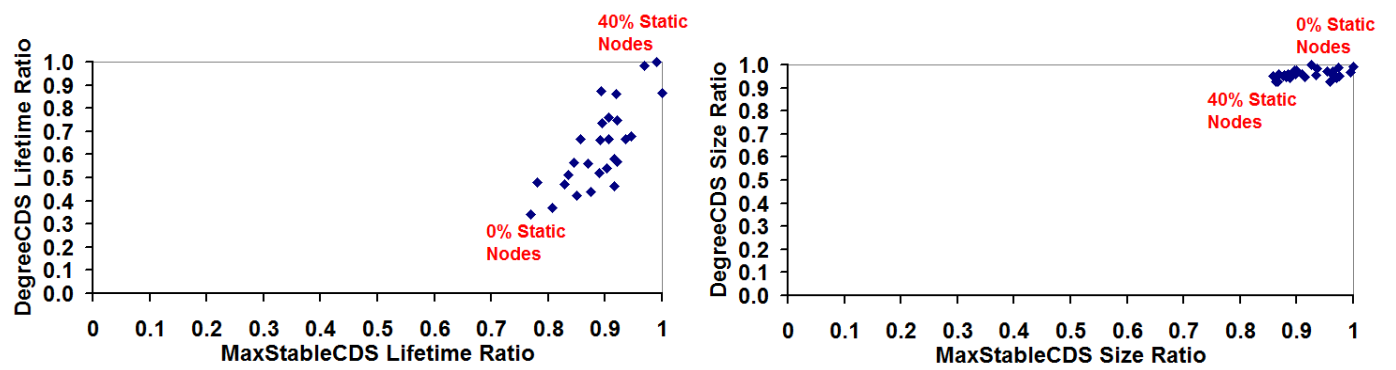

Figure 20. Maximum Stable CDS vs. Degree CDS: Variation in Lifetime and Node Size $\quad\left[v_{\max }=5 \mathrm{~m} / \mathrm{s}\right]$
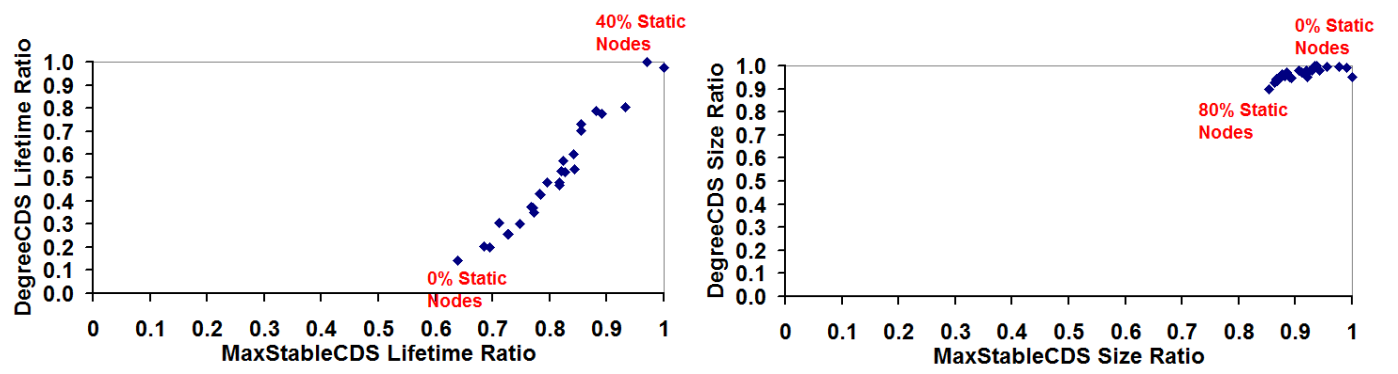

Figure 21. Maximum Stable CDS vs. Degree CDS: Variation in Lifetime and Node Size $\quad\left[v_{\max }=25 \mathrm{~m} / \mathrm{s}\right]$
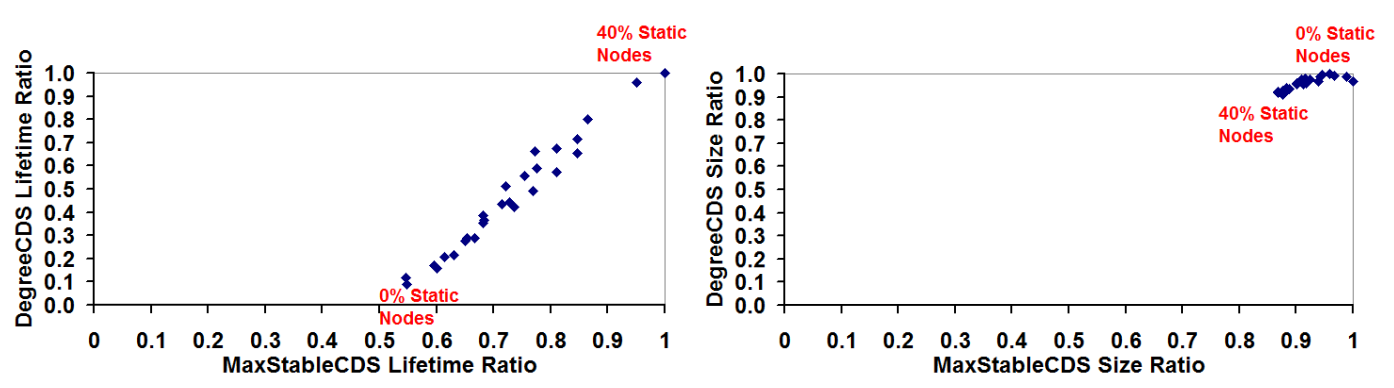

Figure 22. Maximum Stable CDS vs. Degree CDS: Variation in Lifetime and Node Size $\quad\left[v_{\max }=50 \mathrm{~m} / \mathrm{s}\right]$

We could infer that for both the Maximum Stable CDS and Degree-based CDS, the variation in the CDS Lifetime incurred across the simulation scenarios increases with increase in the maximum node velocity; the variation is much more wide-spread in the case of Degree-based CDS (the ratios range all the way from 0.1 to 1.0 in scenarios of moderate and high node mobility). We also observe the ratios for the two CDS strategies to be more arbitrarily distributed in low node mobility scenarios, whereas the ratios incurred for the moderate and high node mobility scenarios seem to exhibit a positive correlation (i.e., as the ratio for the Maximum Stable CDS Lifetime increases, the ratio for the Degree-based CDS Lifetime increases and vice-versa). With regards to the ratio for the CDS Node Size, we observe both the Maximum Stable CDS and Degree-based CDS to sustain only minor variations; the Maximum Stable CDS incurs relatively higher variation compared to the Degree-based CDS, especially with increase in the maximum node velocity. As observed in Section 4.2, the average CDS Node Size for the Maximum Stable CDS decreases with increase in maximum node velocity, and the above said variation could be also attributed towards this factor. 


\section{RELATED WORK}

MANET simulation studies have typically used the Random Waypoint model as the mobility model. As per this mobility model, a mobile node moving in a particular direction could pause for a certain time after reaching a targeted location and then continue to move. The pause time is one of the parameters of the Random Waypoint mobility model. The influence of static nodes on the performance of a communication protocol has been so far evaluated only on the basis of this pause time. Also, such studies have been typically conducted to analyze the performance of unicast routing protocols (e.g., [11]) and multicast routing protocols (e.g., [12]) for MANETs. Even in such works, the focus had been on the impact of pause time on metrics such as delay, throughput, energy consumption, etc, but not explicitly on the lifetime of the communication topologies like paths and trees. To the best of our knowledge, we have not come across any studies that consider the influence of static nodes of any form (including pause time) on the performance of network-wide communication topologies, like that of connected dominating sets for MANETs. In this paper, we chose to designate certain nodes as static throughout the simulation and let the other nodes to move. We wanted to evaluate the impact of operating a MANET with a certain fraction of nodes that are permanently static as well as letting the mobile nodes to pause for awhile every now and then so that the overall fraction of static nodes at any time instant is increased. We conjecture that the observations made in this paper about the impact of static nodes and pause time could help us to choose the appropriate values for these parameters while operating the network at different levels of node mobility.

\section{CONCLUSIONS}

The simulation results confirm our hypotheses: Node pause time has a positive impact on enhancing the stability of the network-wide communication topologies for moderate and high node mobility scenarios; whereas, the presence of a certain fraction of permanently static nodes (nodes that do not move at all) could have a positive impact in enhancing the stability of the network-wide communication topologies in low node mobility scenarios. For a given value of maximum pause time and \% of permanently static nodes, we observe the network to have a relatively larger fraction of static nodes at any time instant when the network is operated is with larger values for the maximum node velocity. As a result, with increase in the maximum node velocity and increase in the pause time of mobile nodes, we observe the lifetime of the Degreebased CDS to approach that of the Maximum Stable CDS. As we simultaneously increase the \% of permanently static nodes as well as the pause time of mobile nodes, we observe the Degreebased CDS to be almost as stable as that of the Maximum Stable CDS. Thus, even if a certain fraction of nodes move faster, the presence of a significant fraction of static nodes helps to boost the lifetime of the Degree-based CDS. In this paper, the Degree-based CDS heuristic did not directly take into account the presence of static nodes while considering nodes for inclusion in the CDS. With the results observed in this paper, we anticipate that if the heuristic is adapted to take into account both the node velocity (or some form of node mobility or non-mobility) and node degree while forming a CDS, the stability of such CDSs could be significantly higher than that incurred with CDSs determined using a degree-based heuristic without any appreciable increase in the CDS node size. We thus anticipate the results of this paper to open new avenues of research for improving the stability of degree-based heuristics for MANETs. 


\section{REFERENCES}

[1] C. Siva Ram Murthy and B. S. Manoj, Ad Hoc Wireless Networks: Architectures and Protocols, Prentice Hall, 1st edition, March 2012.

[2] D. B. Johnson and D. A. Maltz, "Dynamic Source Routing in Ad Hoc Wireless Networks," Mobile Computing - The Kluwer International Series in Engineering and Computer Science, vol. 353, pp. 153-181, 1996.

[3] C. E. Perkins and E. M. Royer, "Ad Hoc On-demand Distance Vector Routing," Proceedings of the 2nd IEEE Workshop on Mobile Computing Systems and Applications, pp. 90-100, New Orleans, LA, USA, Feb 25-26, 1999.

[4] C. W. Wu and Y. C. Tay, "AMRIS: A Multicast Protocol for Ad hoc Wireless Networks," Proceedings of the IEEE Military Communications Conference, vol. 1, pp. 25-29, Oct 31-Nov 3, 1999.

[5] A. B. Mnaouer, L. Chen, C. H. Foh and J. W. Tantra, "OPHMR: An Optimized Polymorphic Hybrid Multicast Routing Protocol for MANET," IEEE Transactions on Mobile Computing, vol. 6, no. 5, pp. 551-562, May 2007.

[6] F. Dai and J. Wu, "Performance Analysis of Broadcast Protocols in Ad Hoc Networks Based on SelfPruning," IEEE Transactions on Parallel and Distributed Systems, vol. 15, no. 11, pp. 1-13, November 2004.

[7] S. Saha, S. R. Hussain and A. K. M. Ashikur Rahman, "RBP: Reliable Broadcast Protocol in Large Scale Mobile Ad Hoc Networks," Proceedings of the 24th IEEE International Conference on Advanced Information Networking and Applications, pp. 526-532, Perth, WA, USA, April 20-23, 2010.

[8] T. H. Cormen, C. E. Leiserson, R. L. Rivest and C. Stein, Introduction to Algorithms, 3rd ed., MIT Press, July 2009.

[9] N. Meghanathan and A. Farago, "On the Stability of Paths, Steiner Trees and Connected Dominating Sets in Mobile Ad Hoc Networks," Ad Hoc Networks, vol. 6, no. 5, pp. 744-769, July 2008.

[10] C. Bettstetter, H. Hartenstein and X. Perez-Costa, "Stochastic Properties of the Random-Way Point Mobility Model,” Wireless Networks, vol. 10, no. 5, pp. 555-567, September 2004.

[11] S. R. Das, C. E. Perkins, E. M. Royer, "Performance Comparison of Two On-demand Routing Protocols for Ad hoc Networks," Proceedings of the IEEE International Conference on Computers and Communications, vol. 1, pp. 3-12, Tel Aviv, Israel, March 26-30, 2000.

[12] K. Kavitha and K. Selvakumar, "Analyzing Multicast Routing Protocols with Different Mobility Models," International Journal of Emerging Technology and Advanced Engineering, vol. 3, no. 1, pp. 33-41, January 2013.

[13] N. Meghanathan, "Exploring the Stability-Energy Consumption-Delay-Network Lifetime Tradeoff of Mobile Ad Hoc Network Routing Protocols," Journal of Networks, vol. 3, no. 2, pp. 17-28, February 2008.

[14] N. Meghanathan, "A Unicast MANET Routing Protocol to Simultaneously Minimize the StabilityHop Count Tradeoff and End-to-End Delay," Proceedings of the 9th International Conference on Information Technology : New Generations, pp. 60-64, Las Vegas, NV, USA, April 16-18, 2012.

[15] N. Meghanathan, "Benchmarks and Tradeoffs for Minimum Hop, Minimum Edge and Maximum Lifetime per Multicast Tree in Mobile Ad hoc Networks," International Journal of Advancements in Technology, vol. 1, no. 2, pp. 234-251, October 2010.

\section{Author}

Dr. Natarajan Meghanathan is a tenured Full Professor of Computer Science at Jackson State University, USA. His areas of research interests are Network Science and Graph Theory, Wireless Ad hoc Networks and Sensor Networks, Cyber Security and Machine Learning. He has published more than 150 peer-reviewed articles and obtained grants from several federal agencies. He serves as the editor-in-chief of three international journals as well as serves in the organizing committees of several international conferences.

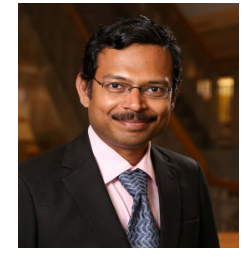

